\title{
Robust Particle Filtering for Object Tracking
}

\author{
Daniel Rowe, Ignasi Rius, Jordi Gonzàlez, and Juan J. Villanueva \\ Computer Vision Centre/Department of Computer Science, \\ Universitat Autònoma de Barcelona, 08193 Bellaterra, Barcelona, Spain \\ drowe@cvc.uab.es
}

\begin{abstract}
This paper addresses the filtering problem when no assumption about linearity or gaussianity is made on the involved density functions. This approach, widely known as particle filtering, has been explored by several previous algorithms, including Condensation. Although it represented a new paradigm and promising results have been achieved, it has several unpleasant behaviours. We highlight these misbehaviours and propose an algorithm which deals with them. A test-bed, which allows proof-testing of new approaches, has been developed. The proposal has been successfully tested using both synthetic and real sequences.
\end{abstract}

\section{Introduction}

The increasing interest in tracking is motivated by the huge number of promising applications that can now be tackled in real time thanks to recent technological advances. These applications include performance analysis of human motion, surveillance, video-indexing and smart interfaces, among others.

However, tracking can be extremely complex and time-consuming. In the first place, strong requirements are mandatory. Real-time processing, extreme robust performances or high accuracy may be critical. Moreover, several difficulties must be expected such as multiple-target tracking with unknown and highly non-linear dynamics, presence of heavy background clutter, strong noise and partial occlusions.

This paper focuses on tracking by means of particle-filtering in the conditions described above. This approach has been explored by several previous algorithms, including Condensation [3. Although some results were achieved, Condensation-based algorithms have several unpleasant behaviours. In this paper, we highlight these misbehaviours and propose an algorithm which deals with them, also in presence of the above-presented tracking difficulties.

The remainder of this paper is organized as follows. Section 2 covers the probabilistic framework and revises Condensation, exposing its misbehaviours. Section 3 describes the proposed algorithm. Section 4 shows experimental results with both synthetic and real sequences. Section 5 concludes this paper.

\section{Particle-Filtering Background}

A probabilistic framework is commonly used as a way to perform tracking [5]. Classical approaches, such as the Kalman Filter, rely on linearity and gaussian- 
ity assumptions about the involved distributions. More recent works make use of Bayesian filters combined with Monte Carlo Simulation methods in order to deal with nonlinear and non-Gaussian transition and sensor models [12]. However, these methods present several important drawbacks. A great number of improvements have been introduced in recent years [76] but there is still much ground to cover before solving unconstrained tracking.

We are interested in filtering, that is, the computation of the belief state $\mathbf{S}_{t}$ given all evidence to date $\mathbf{e}_{1: t}$. Posteriors are calculated through recursive estimation:

$$
P\left(\mathbf{S}_{t} \mid \mathbf{e}_{1: t}\right)=\underbrace{P\left(\mathbf{e}_{t} \mid \mathbf{S}_{t}\right)}_{\text {updating }} \int \underbrace{P\left(\mathbf{S}_{t} \mid \mathbf{s}_{t-1}\right)}_{\text {prediction stage }} \underbrace{p\left(\mathbf{s}_{t-1} \mid \mathbf{e}_{1: t-1}\right)}_{\text {likelihood }} d \mathbf{s}_{t-1} .
$$

This pdf is projected forward according to the transition model, making a prediction, and it is updated in agreement with the likelihood function value based on the new evidence.

Unfortunately, recursive estimation leads to expressions that are impossible to evaluate analytically unless strong assumptions are applied. This problem is overcome by simulating $N$ i.i.d. samples which approximate the posterior pdf. This approach is known as particle filtering within the control field and survival of the fittest in artificial intelligence. Such methods, renamed as Condensation [3], were first introduced in the computer vision research area by Isard and Blake.

The method works as follows: the posterior pdf at time $t-1$ is represented by a set of particles or samples, $\left\{\mathbf{s}_{t-1}^{i} ; i=1: N\right\}$. The temporal $\operatorname{prior}\left\{\hat{\mathbf{s}}_{t}^{i}\right\}$ is obtained by applying the transition model $P\left(\mathbf{S}_{t} \mid \mathbf{s}_{t-1}\right)$ to each sample. The likelihood $P\left(\mathbf{e}_{t} \mid \mathbf{S}_{t}\right)$ is represented by weights $\pi_{t}^{i}$ according to the likelihood values at the sample points. Once all samples have been propagated and measured, the set is re-sampled using normalized weights $\bar{\pi}_{t}^{i}$ as probabilities. The sample set $\left\{\mathbf{s}_{t}^{i} ; i=1: N\right\}$ represents the posterior at time $t$. Expectations can be approximated as:

$$
\mathbb{E}_{P\left(\mathbf{S}_{t} \mid e_{1: t}\right)}\left(\mathbf{S}_{t}\right) \simeq \sum_{i=1}^{N} \bar{\pi}_{t}^{i} \hat{\mathbf{s}}_{t}^{i} \simeq \frac{1}{N} \sum_{i=1}^{N} \mathbf{s}_{t}^{i} .
$$

However, Condensation has several unpleasant behaviours as stated in [4]. Sampling impoverishment is one of the main drawbacks of re-sampling algorithms. Samples are spread around several modes indicating hypotheses in the state space. Nevertheless, some of them are spurious. Similarly to genetic drift, there is a non-negligible probability of losing modes, a low probability of recovering them and the remaining modes could be all spurious. It can also be derived from this fact that different runs of the algorithm may lead to different results. Therefore, computed expectations in different runs have high variance although computed expectations within the same algorithm run have low variance, making the tracker look stable. In addition, the sample set size $N$ is kept constant over 
time and there is no information about how large $N$ should be for a requested precision. Once $N$ has been heuristically set, it may happen that at later times larger values of $N$ may be required. Finally, Condensation was designed to keep multiple hypotheses, but only for a single target.

\section{Towards Robust Tracking}

We propose an algorithm based on particle filtering. In contrast with Condensation, in which the target is represented by means of contours, we rely on a pixel-wise approach since it is expected to be more robust to partial occlusions.

The motion of the centre of a bounding box is modelled using first-order dynamics in image coordinates. The l-labeled target's state is defined as $\mathbf{s}_{t}^{l}=$ $\left(\mathbf{x}_{t}^{l}, \mathbf{u}_{t}^{l}, \mathbf{w}_{t}^{l}, \mathbf{A}_{t}^{l}\right)^{T}$. Each component represents the position, speed, bounding-box size and appearance, respectively. A label associates one specific appearance model to the corresponding samples, allowing multiple-target tracking.

\subsection{Likelihood Function}

The likelihood function gives the pdf of image features given the state. The selected features are pixel-oriented. Thus, the appearance will be given by a matrix whose elements are the pixel intensity values. Therefore, it can be assumed that the appearance is independent from the speed component. Let $\mathbf{I}_{t}$ be a matrix whose elements are the scene pixel intensity values at time $t$. Given the predicted position $\mathbf{x}_{t}=(x, y)$ and bounding-box size $\mathbf{w}_{t}=(w, h)$, the corresponding image subregion is denoted by $\mathbf{I}_{t}^{p}$. The model appearance matrix must be scaled according to the sample size. Let $\mathbf{A}_{t}^{s}$ be the scaled matrix for the model. Considering a smooth process, we assume that the appearance is constant between frames. Assuming also White Additive Gaussian Noise (WAGN), the likelihood function can be expressed as:

$$
\begin{aligned}
P\left(\mathbf{I}_{t} \mid \mathbf{S}_{t}\right) & =P\left(\mathbf{I}_{t}^{p} \mid \mathbf{A}_{t}^{s}\right) \\
& =\frac{1}{M} \sum_{a, b \in \mathbf{A}_{t}^{s}} \mathcal{N}\left(\mathbf{I}_{t}^{p}(a, b) ; \mathbf{A}_{t}^{s}(a, b), \sigma_{a}^{2}\right) .
\end{aligned}
$$

where $M$ is the number of pixels of the appearance model, $(a, b)$ defines a pixel position in the appearance matrix $\mathbf{A}_{t}^{s}$ and $\sigma_{a}^{2}$ is the estimated camera noise variance, which randomly influences the pixel intensity values.

\subsection{Weight Normalization}

Multiple-target tracking causes several problems including that the target with higher likelihood may monopolize the sample set. If data association is feasible, using a prior density to generate new samples reduces the risk of sampling impoverishment. However, this is not always possible, specially during occlusions or while the target is surrounded by background clutter. In this case, those targets whose samples exhibit lower likelihood have higher probability of being lost, 
since the probability of propagating one mode is proportional to the cumulative weights of the samples that constitute it.

Two kind of modes can be distinguished: samples with different labels belong to different modes, and thereby, several targets can be tracked simultaneously; secondly, samples with the same label could be spread around different modes. This fact allows us to keep several hypotheses for a single target; hopefully, one of them represents the true target state and the others are due to background clutter. In order to avoid single-target modes absorbing other target samples, genetic drift must be prevented. This fact happens due to the lack of genetic memory: we propose to include a memory term which takes into account the number of targets being tracked. Hence, weights are normalized according to:

$$
\bar{\pi}_{t}^{i, l}=\frac{\pi_{t}^{i, l}}{\sum_{i=1}^{N} \pi_{t}^{i, j}} \frac{1}{L}, \text { where } j=l,
$$

where $L$ is the number of targets being tracked. It does not assign a fixed number of samples to each target but ensures that each target will have the same probability of being propagated.

On the other hand, modes due to clutter are pruned because of differences in their dynamics. It is unlikely that any sample tracks local clutter since it implies highly abrupt changes in the dynamics. Thus, non-loss of the true mode depends on how the different hypotheses are generated.

\subsection{Sample Dynamics}

Since our likelihood function depends on the sample position but does not depend on its speed, propagated samples could have a small position error, but their speed values could become completely different from the true one in a few frames. Targets could be tracked since we are in a multiple-hypothesis scenario, but an important proportion of samples will be wasted.

Thus, we feed back the estimated target speed at time $t-1$, denoted as $\mathbf{u}_{t-1}^{l}$, into the prediction. Subsequently, speeds are not estimated from the selected samples, but calculated according to the history of positions. Both position and speed estimation are regularised according to their corresponding histories. Thus, predictions are computed as follows:

$$
\hat{\mathbf{x}}_{t}^{i, l}=\mathbf{x}_{t-1}^{i, l}+\mathbf{u}_{t-1}^{i, l} \Delta_{t}+\xi_{\mathbf{x}}^{i, l}, \quad \hat{\mathbf{u}}_{t}^{i, l}=\mathbf{u}_{t-1}^{l}+\xi_{\mathbf{u}}^{i, l} .
$$

The random terms $\xi_{\mathbf{x}}^{i, l}, \xi_{\mathbf{u}}^{i, l}$ provide the system with a diversity of hypotheses and, subsequently, samples with high likelihood are more probably propagated. The $l$-target position and speed are estimated according to:

$$
\begin{aligned}
& \mathbf{x}_{t}^{l}=\left(\mathbf{x}_{t-1}^{l}+\mathbf{u}_{t-1}^{l} \Delta_{t}\right)\left(1-\alpha_{\mathbf{x}}\right)+\left(\frac{1}{N_{l}} \sum_{i=1}^{N} \bar{\pi}_{t}^{i, l} \hat{\mathbf{x}}_{t}^{i, l}\right) \alpha_{\mathbf{x}}, \\
& \mathbf{u}_{t}^{l}=\mathbf{u}_{t-1}^{l}\left(1-\alpha_{\mathbf{u}}\right)+\left(\mathbf{x}_{t}^{l}-\mathbf{x}_{t-1}^{l}\right) \alpha_{\mathbf{u}},
\end{aligned}
$$

where $\alpha_{\mathbf{x}}, \alpha_{\mathbf{u}}$ denote the adaptation rates and $N_{l}$ the number of samples of the $l$-target. The speed is fed back when predicting the following sample state. 


\subsection{Sample Size Propagation}

The target dimensions are predicted using a random-walk model:

$$
\hat{\mathbf{w}}_{t}^{i, l}=\mathbf{w}_{t-1}^{i, l}+\xi_{\mathbf{w}}^{i, l},
$$

where $\xi_{\mathbf{w}}^{i, l}$ is a random term obtained according to the target's size covariance. Sample likelihoods are calculated as the mean of the probabilities of belonging to the target of each pixel within the bounding box. Thus, it depends on the normalised overlapping area $A_{o}$ between the target's bounding-box and a misaligned sample one, given by:

$$
A_{o}=1-\frac{1}{w} \Delta x-\frac{1}{h} \Delta y+\frac{\Delta x * \Delta y}{w * h} .
$$

Thus, the effects of misalignments depend on the target's size: bigger targets tolerate bigger shifts without significant likelihood falls. We are interested in the relation between the likelihoods of two particles since, once all weights are normalised, the probability of choosing one particle instead of other depends on this relation. This relation must make the 'aligned' sample likely enough to be re-sampled instead of the misaligned one. This is achieved by mapping sample likelihoods using monomial functions. Exponents are selected on-line, according to the target size, ensuring a minimum slope of the overlapping area.

On the other hand, those samples with smaller sizes are expected to have higher likelihoods since a likelihood computation involves a normalization according to the sample size and smaller samples can have relative bigger overlapping regions. As it is empirically proved, samples tend to smaller sizes and, eventually, collapse. These results strongly suggest that higher bounding boxes should be slightly favoured in the re-sampling process. Therefore, a term related to the relative sample sizes is included in the likelihood computation.

\section{Experimental Results}

The performance of the algorithm has been tested using both synthetic and real data. Synthetic data allow us to achieve two goals: we have access to the ground truth, therefore deviations and performance can be accurately measured;
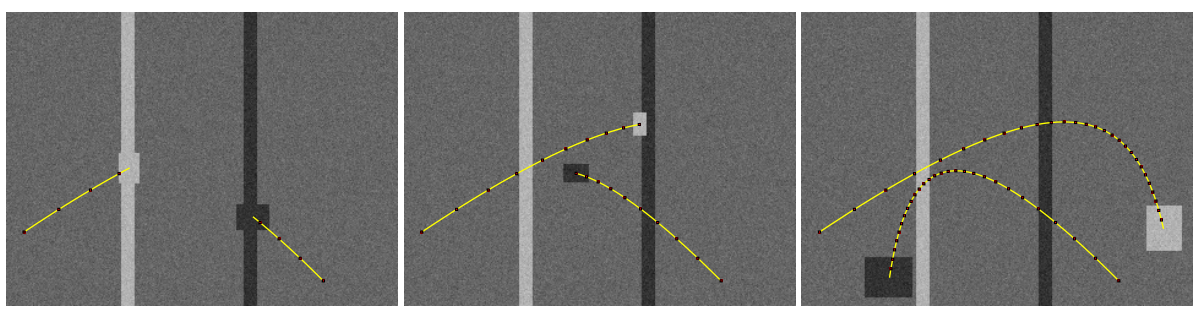

Fig. 1. Ground Truth 
in addition, experimental conditions can get harder and harder on each aspect independently, thereby maximum performances can be measured.

A two-moving-target synthetic experiment has been designed. It covers several difficulties a tracker can run into, see Fig. 1. The background pixel intensity values follow a normal distribution. Both targets' pixel intensity values also have a normal distribution around different means. Two vertical strips are drawn in the background, simulating heavy clutter. Their distributions are identical to those of both targets, thereby mimicking them. Strong acquisitiondevice noise, modelled as WAGN, is simulated. A highly non-linear dynamic is considered: both targets move as projectiles which are shot into an environment with gravity and air friction. Each target constantly reduces its width and height during the first third of the experiment, and increases them during the rest of it. Thus, areas fluctuate from 209 to 1435 pixels and aspect ratios from 1.7 to 1.3 . Tracking is performed over $T=300$ frames using $N=100$ samples.

Without the proposed weight normalization, the tracker loses a target due to the lack of samples, see Fig. 2. (a). On the other hand, after the proposed weight normalization, the mean number of samples per target in each run fluctuates between $49.5 \%$ and $50.5 \%$, see Fig. 2.(b).
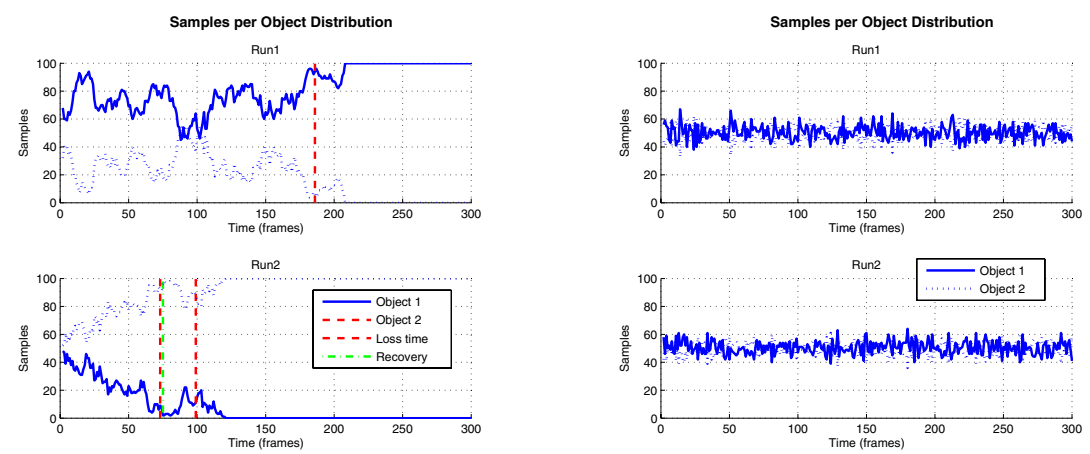

(a) Without proposed weigh normalisation

(b) After applying the normalisation

Fig. 2. Samples per object distribution

Table 1. Mean position error

\begin{tabular}{|c||c|c|}
\hline \multicolumn{3}{|c|}{ Mean normalized error } \\
\hline \hline & Target 1 & Target 2 \\
\hline Run 1 & 0.1163 & 0.1309 \\
\hline Run 2 & 3.8864 & 0.1182 \\
\hline Run 3 & 0.1222 & 0.1226 \\
\hline Run 4 & 0.1101 & 2.4679 \\
\hline
\end{tabular}

\begin{tabular}{|l||c|c|}
\hline \multicolumn{3}{|c|}{ Mean normalized error } \\
\hline \hline & Target 1 & Target 2 \\
\hline \hline Run 1 & 0.0715 & 0.0716 \\
\hline Run 2 & 0.0849 & 0.1163 \\
\hline Run 3 & 0.0987 & 0.1289 \\
\hline Run 4 & 0.0645 & 0.0595 \\
\hline
\end{tabular}

(a) Without the speed feed-back

(b) After feeding the speed back 


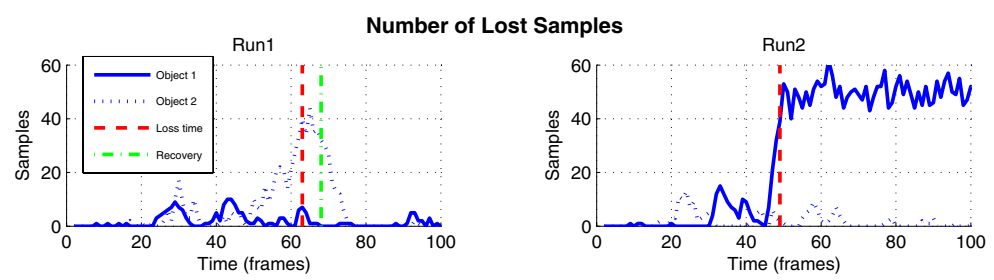

Fig. 3. Evolution of lost samples without feeding the speed back
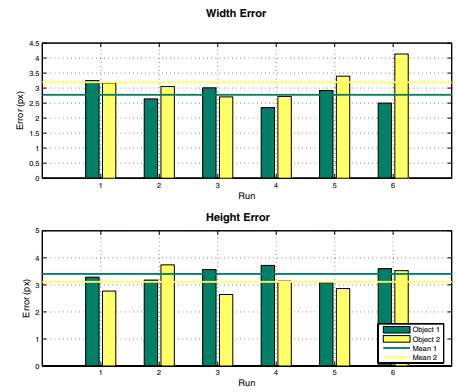

Fig. 4. Mean size error

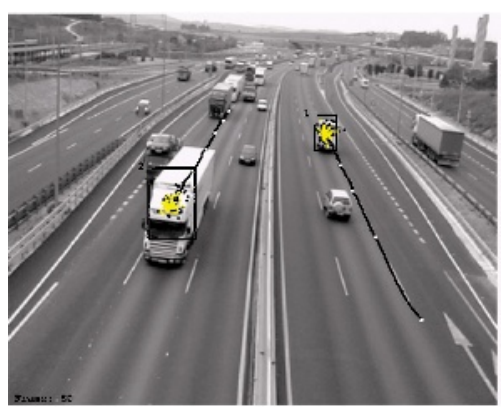

(a)

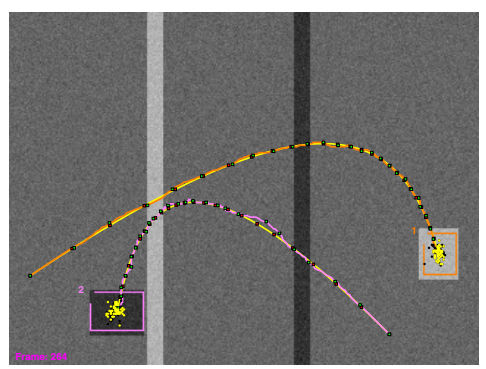

Fig. 5. Target performance

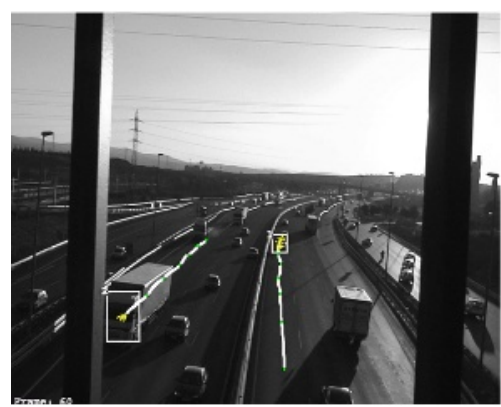

(b)

Fig. 6. Target performance on real sequences

Table 1.(a) shows the mean normalized error, according to the target size, in the estimation of the target position without regularising and feeding the speed back, whereas Table1.(b) shows the same results after applying it. A significant error reduction can be appreciated besides the fact that now none of the targets is ever lost. Fig. 3 shows the number of samples that had lost their target without our proposed speed feed-back and estimation. After applying it, the number of lost samples is negligible. The error in estimating the target size is shown in Fig. 4. Six runs for both targets are represented. 
The tracker performance is shown in Figs. 5, 6. Fig. 5 presents one synthetic run; Fig. 6. (a) shows a traffic sequence; Fig. 6. (b) exhibits a traffic sequence under heavy shadow and reflectance conditions.

\section{Conclusions}

We have proposed a particle-filter based algorithm. It deals with multiple-target tracking whose dynamics are highly non-linear. They move through an environment with complex clutter, which mimics the target appearances, and strong noise. A new approach is taken to deal with one of Condensation's great misbehaviours, the sampling impoverishment. This problem becomes critical in a multiple target-tracking scenario. The proposed sample-weight normalization prevents from the loss of any of the targets due to the lack of samples. The dynamics updating is set by feeding the estimated speed back into the prediction stage. The speed is not estimated according to the sample speeds since the likelihood does not provide speed measures. Instead, it is estimated from successive position estimations. Subsequently, both target's position and speed are regularised. Sample wastage is significantly reduced. The tracker has been successfully tested in both synthetic and real experiments concerning traffic surveillance. They are currently being applied in real applications relative to people tracking. Encouraging results are being achieved. Future research will be focused on colour-based likelihoods.

Acknowledgments. This work has been supported by the Spanish CICYT TIC 2003-08865 and the Generalitat de Catalunya Research Department (DURSI).

\section{References}

1. S. Arulampalam, S. Maskell, N. Gordon, and T. Clapp. A tutorial on particle filters for on-line non-linear/non-gaussian bayesian tracking. Tran. on Signal Processing, $50(2): 174-188,2002$.

2. A. Doucet. On sequential simulation-based methods for bayesian filtering. Technical Report TR310, Cambridge University, 1998.

3. M. Isard and A. Blake. Condensation - conditional density propagation for visual tracking. International Journal of Computer Vision, 29(1):5-28, 1998.

4. O. King and D. A. Forsyth. How does CONDENSATION behave with a finite number of samples? In 6th ECCV, Dublin, Ireland, volume 1, pages 695-709, 2000.

5. R. Russell and P. Norvig. Artificial Intelligence, a Modern Approach, chapter 13-15. Prentice Hall, second edition, 2003.

6. R. van der Merwe, N. de Freitas, A. Doucet, and E. Wan. The Unscented Particle Filter. Technical Report TR380, Cambridge University, 2000.

7. X. Varona, J. Gonzàlez, X. Roca, and J.J. Villanueva. iTrack: Image-based Probabilistic Tracking of People. In 15th ICPR, Barcelona, Spain, volume 3, pages 1110-1113, 2000. 